\title{
Zero-loss Filtered TEM Imaging Of Microelectronic Structures Using In-Column Filter Technology
}

\author{
H. Stegmann, G. Benner \\ Carl Zeiss NTS GmbH, Carl-Zeiss-Str. 56, 73447 Oberkochen, Germany
}

In semiconductor manufacturing, conventional TEM imaging is routinely used for cross-sectional characterization of the smallest structures of microelectronic products. Despite the use of technically mature focused ion beam (FIB) thinning techniques for high quality site-specific TEM specimen preparation, optimum specimen thickness cannot always achieved. In this paper, the benefits of zero-loss (ZL) filtering for imaging of typical samples at different specimen thicknesses will be discussed.

For the examples shown in Figs. 1-3, two FIB-cut TEM samples were prepared from a test structure of a fully processed microprocessor. One lamella was thinned to the optimum thickness for conventional TEM imaging of such structures of about $80 \mathrm{~nm}$, the other one was thinned to about $200 \mathrm{~nm}$ only. A Zeiss LIBRA200 energy-filtering TEM with in-column corrected $90^{\circ}$ Omega filter, operated at $200 \mathrm{kV}$, was used for recording ZL filtered and unfiltered images from shallow trench isolation (STI) areas (Figs. 1 and 2), and from copper interconnect vias (Fig. 3). Imaging conditions were always identical, except for the insertion of an energy selecting slit aperture for ZL filtering.

A clear contrast improvement by ZL filtering was observed for the thin sample (Fig. 1). For the thick sample, the ZL filtered images show a significant improvement in image quality and visibility of critical structures (Figs. 2 and 3). Intensity profiles across the tantalum barrier layer at the bottom of the via indicate a better definition of the tantalum/copper interfaces in the ZL filtered image (Fig. 3 , right). The thickness of the barrier layer can thus be measured more accurately.

Precise feature size measurement and short turnaround time are essential requirements to TEM imaging in large-scale semiconductor production [1]. ZL filtering removes blurring that is caused by inelastic scattering, and introduces additional filter contrast [2]. The interpretability of the images is improved, resulting in more accurate measurement of critical structure dimensions. The usable sample thickness range is expanded. Thus, additional time-consuming FIB thinning of specimens that were initially cut too thick can in many cases be avoided. Furthermore, in a TEM equipped with an in-column energy filter, ZL filtered imaging is the standard imaging mode and does not involve additional operational effort.

Therefore, ZL filtered TEM imaging using an in-column energy filter offers significant advantages over conventional TEM imaging for structural characterization of microelectronic products at high sample throughput, especially regarding future semiconductor manufacturing process technology generations with further reduced structure dimensions.

\section{References}

[1] H.J. Engelmann et al., Pract. Metallography 39 (2002) 117.

[2] I. Angert et al., Ultramicr. 81 (2000) 203.

[3] Supply of TEM samples by E. Zschech and H.J. Engelmann (AMD Saxony LLC \& Co. KG) is gratefully acknowledged. 

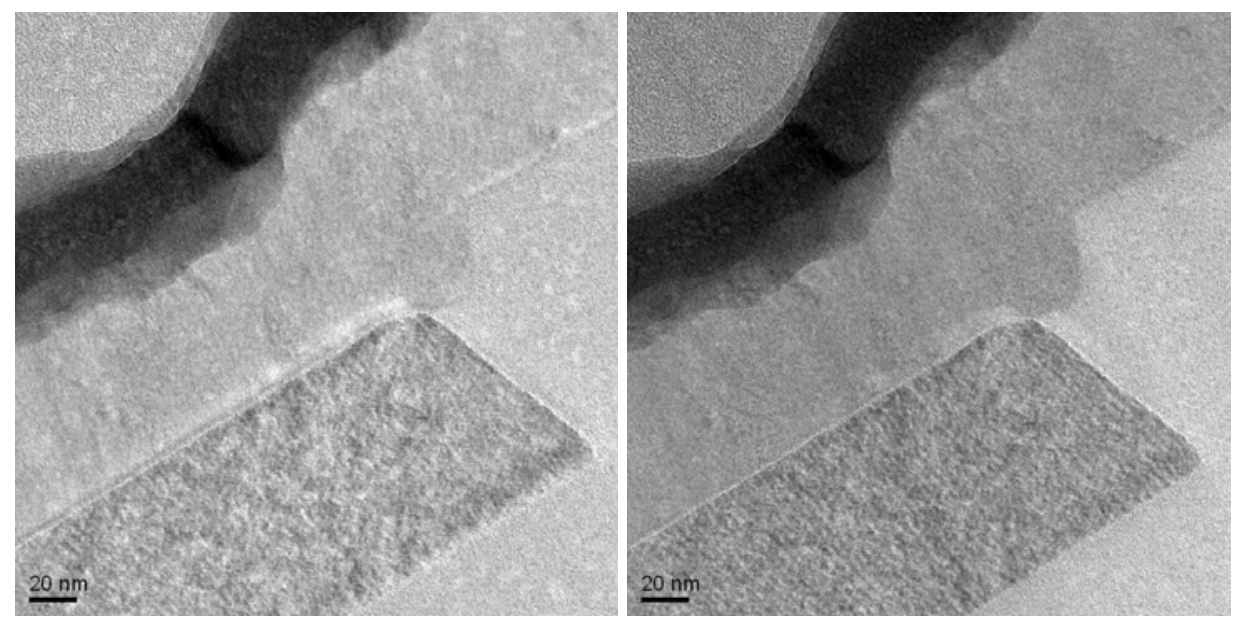

FIG. 1. STI corner, unfiltered (left) and ZL filtered (right). Specimen thickness about $80 \mathrm{~nm}$.
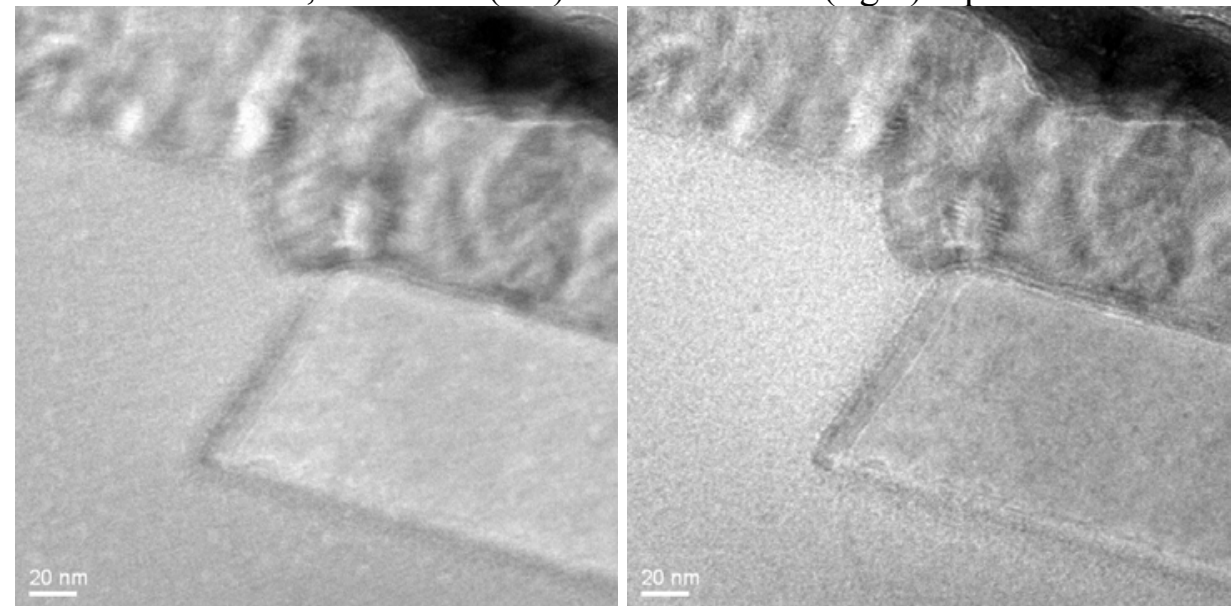

FIG. 2. STI corner, unfiltered (left) and ZL filtered (right). Specimen thickness about $200 \mathrm{~nm}$.
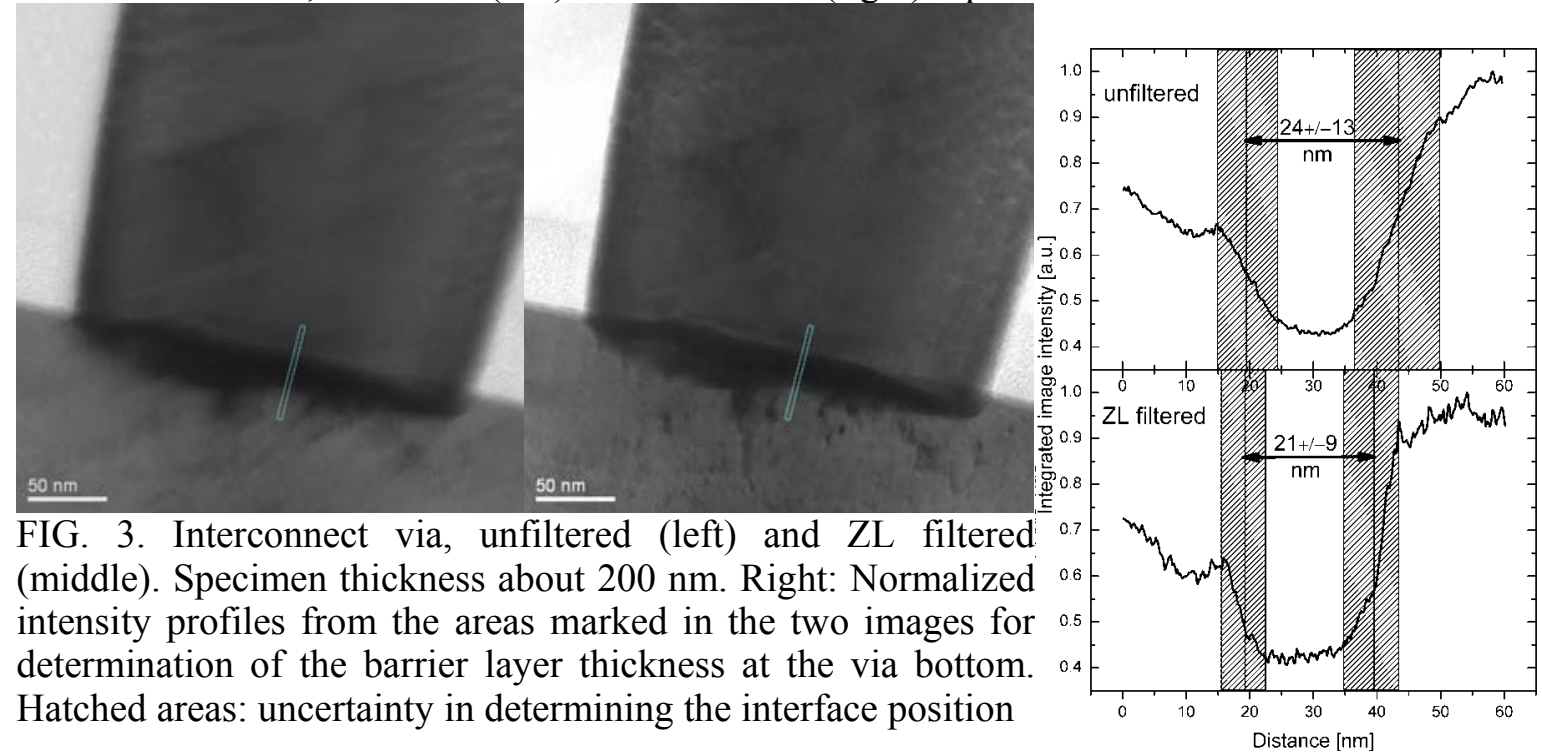

FIG. 3. Interconnect via, unfiltered (left) and ZL filtered (middle). Specimen thickness about $200 \mathrm{~nm}$. Right: Normalized intensity profiles from the areas marked in the two images for determination of the barrier layer thickness at the via bottom. Hatched areas: uncertainty in determining the interface position 